\title{
Bullying e abuso sexual em adolescentes: uma revisão integrativa
}

\section{Bullying and sexual abuse in adolescents: an integrative review}

\section{Thiago de Oliveira Felizmino' ${ }^{1}$ (D) Joilson Pereira da Silva² (D)}

${ }^{1}$ Autor para correspondência. Universidade Federal de Sergipe (Aracaju). Sergipe, Brasil. thiagofelizmino@gmail.com ${ }^{2}$ Universidade Federal de Sergipe (Aracaju). Sergipe, Brasil. joilsonp@hotmail.com

RESUMO | OBJETIVOS: Este estudo é uma revisão integrativa que objetivou reunir as principais discussões associando adolescentes e situações de bullying e/ou abuso sexual, publicadas entre 2008-2018. MÉTODOS: Foram utilizadas as bases: SciELO, PsycINFO, Scopus e PePSIC. Ao final, 21 estudos foram selecionados para as análises. RESULTADOS: Os principais resultados demonstraram que, em nível quantitativo, estudos sobre o abuso sexual se sobressaíram em relação aqueles que investigaram o bullying. CONSIDERAÇõES FINAIS: Em razão de apenas 1 estudo ter associado numa mesma pesquisa ambos os fenômenos, sugere-se o investimento de pesquisas deste nível entre adolescentes no contexto escolar.

PALAVRAS-CHAVE: Bullying. Abuso sexual. Adolescência.
ABSTRACT | OBJECTIVES: This study is an integrative review that aimed to gather the main discussions associating adolescents and bullying and / or sexual abuse cases, published between 2008-2018. METHODS: The bases were used: SciELO, PsycINFO, Scopus, and PePSIC. In the end, 21 studies were selected for the analyzes. RESULTS: The main results showed that, on a quantitative level, studies on sexual abuse outperformed those who investigated bullying. FINAL REMARKS: Because only one study has associated both phenomena in the same research, it is suggested to invest in research of this level among adolescents in the school context.

KEYWORDS: Bullying. Sexual abuse. Adolescence. 


\section{Introdução}

A violência na adolescência não é uma questão que diz respeito apenas à família das pessoas envolvidas, mas constitui-se em grave problema de saúde públi$\mathrm{ca}$, que deve ser observado em todos os contextos onde os jovens estão inseridos (Braga \& Dell' Aglio, 2012). Neste sentido, o bullying e o abuso sexual, depois da negligência e das violências físicas, se destacam como sendo os subtipos de violências (intrafamiliar, extrafamiliar, institucional e escolar) em que os adolescentes estão mais susceptíveis (Fontes et al., 2017; Landini, 2011).

O bullying, mais especificamente dentro do ambiente escolar, trata-se da hostilidade que parte de um aluno mais velho ou mais forte (ou até mesmo grupo), de modo intencional e frequente, em direção a um alvo considerado frágil (aluno com características específicas: físicas, a saber, gordo, magro, alto ou baixo; com deficiência sensorial e/ou intelectual; socioeconômicas; étnicas; orientação sexual, etc.) (Antunes \& Zuin, 2008; Fante, 2005). Em outras palavras, é caracterizado através dos seguintes aspectos: a) intencionalidade do dano por parte do autor; b) intimidações de ordem física, verbal e/ou psicológica; c) periodicidade dos atos violentos; d) o desencadeamento de efeitos negativos em quem foi alvo de bullying; e) o autor recebe apoio de terceiros; f) o alvo, além de não ser o provocador das agressões, encontra-se totalmente indefeso; g) presença hierárquica de dominação e submissão entre autor e alvo (Musitu et al., 2009; Souza, 2013).

Enquanto que, de acordo com o dicionário de psicologia da Associação Americana de Psicologia [APA] (2010), o abuso sexual se configura como sendo a prática sexual forçada (contra a vontade e/ou sem o consentimento do indivíduo). Também estão inclusos jogos sexuais, com ou sem penetração sexual, com um indivíduo incapaz de dar o consentimento devido a prejuízos mentais e/ou por estar abaixo da idade estatutária de consentimento. No tocante às vítimas, podem ser divididas em duas populações: primeira, composta por pessoas mais jovens, vítimas de um agressor conhecido (vizinho, familiar ou responsável), com menor taxa de realização de denúncias e pouca procura por auxílio psicológico; segunda, composta por pessoas agredidas por estranhos, acima dos 20 anos, com maiores taxas de agressão física, que incidem em realizações de denúncias e procuras por auxílio psicológico (Facuri et al., 2013).

Tanto o bullying quanto o abuso sexual, apesar de suas singularidades, são fenômenos desencadeadores de inúmeras consequências semelhantes nos alvos e vítimas. Em relação às pessoas que foram alvo de bullying, diversas pesquisas revelaram o desenvolvimento de dificuldade em se relacionar; depressão; suicídio e/ou homicídio; prejuízos no desenvolvimento; baixo rendimento escolar, absenteísmo e/ou evasão escolar (Chaves \& Sousa, 2018; Fante, 2005; Molcho et al., 2009; Olweus, 1993; Pereira, 2008). Entre as vítimas de abuso sexual também foram encontrados: dificuldades na adaptação interpessoal, de relacionamento com figura masculina, de adaptação sexual e de adaptação afetiva; sentimento de culpa; automutilação; suicídio; depressão; isolamento; despersonalização; transtorno de ansiedade de doença, etc. (Florentino, 2015; Landini, 2011).

Recorrendo à pesquisa nacional de saúde do escolar (PeNSE) realizada com alunos do $9^{\circ}$ ano do ensino fundamental, em todo o Brasil, percebeu-se que a prevalência da prática do bullying gira em torno de $19,8 \%$, com maior ocorrência para o sexo masculino $(24,2 \%)$ em relação ao feminino (15,6\%) (Instituto Brasileiro de Geografia e Estatística [IBGE], 2016). Em relação ao abuso sexual, pesquisa a partir de dados do sistema de vigilância de violências e acidentes (VIVA), demonstrou que no período entre 2010-2014, das 2.226 notificações de violência sexual contra crianças e adolescentes no Brasil, 60,9\% foram caracterizadas como estupro, destacando-se o sexo feminino como o perfil de vítima mais predominante $(63,8 \%)$ (Santos et al., 2018).

Destarte, implementar estudos sobre essas duas temáticas em adolescentes pode auxiliar na compreensão de suas vicissitudes e na promoção do desenvolvimento de relações saudáveis no ambiente familiar, escolar e na própria sociedade, assim como no enfrentamento sistemático por meio de políticas comunitárias e públicas tanto do bullying quanto do abuso sexual. Para tanto, esta revisão integrativa da literatura objetiva reunir as principais discussões associando adolescentes e situações de bullying e/ou abuso sexual publicadas entre 2008-2018. 


\section{Método}

Trata-se de uma revisão integrativa da literatura, por considerar sua finalidade, que é sintetizar resultados obtidos em pesquisas sobre um tema ou questão, de maneira sistemática, ordenada e abrangente, visando obter informações mais amplas sobre um assunto, constituindo, assim, um corpo de conhecimento (Mendes et al., 2008). A pesquisa foi desenvolvida em seis etapas, a saber: a) identificação do tema e questão de pesquisa; b) estabelecimento de critérios para inclusão e exclusão de estudos na literatura; c) definição das informações a serem extraídas dos estudos selecionados; d) avaliação dos estudos incluídos; e) interpretação dos resultados; f) e apresentação da revisão/síntese do conhecimento como considerações finais.

De posse da questão de pesquisa (quais foram as principais discussões associando adolescentes e situações de bullying e/ou abuso sexual publicadas entre 2008-2018?) como estratégia de busca, foram selecionadas as seguintes bases de dados digitais: SciELO, PsycINFO, Scopus e PePSIC, e padronizou-se a utilização dos seguintes descritores e operadores booleanos: bullying OR "sexual abuse" AND adolescence.

As buscas dos descritores foram restringidas ao título e resumo dos artigos. Foram incluídas publicações entre 2008 e 2018 que investigaram a relação entre adolescentes e o bullying e/ou abuso sexual, nas línguas portuguesa $(n=11)$, inglesa $(n=8)$ e espanhola ( $n$ $=2$ ). Foram excluídos artigos incompletos, repetidos, com acessos restritos ou pagos, teses, dissertações, capítulos de livros, resumos, resenhas e ensaios.

Inicialmente, foram identificados 3.787 estudos. Após exclusão dos artigos considerando os critérios de exclusão e inclusão $(n=2.786 ; 73,6 \%)$, foi feita a leitura do título e resumo de 1.001 estudos restantes. Excluíram-se 979 trabalhos $(97,8 \%)$ após essa etapa. A amostra final ficou composta por 21 estudos. As estratégias utilizadas nas respectivas bases de dados e motivos da exclusão seguiram as recomendações
PRISMA [Preferred Reporting Items for Systematic Reviews e Meta-Analysis] (Moher et al., 2009) e foram apresentadas em fluxograma (ver Figura 1).

Após leitura minuciosa de cada um dos artigos selecionados, foram sintetizados aspectos para análises quanto aos países, área de concentração e ano, análises das características amostrais, tipo de estudo e instrumento que analisou o bullying e/ou abuso sexual em adolescentes, e análises dos objetivos e principais resultados dos estudos, oportunidade em que os achados mais relevantes foram categorizados.

\section{Resultados}

Dentre o total de estudos selecionados, $52,4 \%$ em suas pesquisas abordaram o abuso sexual $(n=11$; E1, E2, E5, E6, E7, E8, E9, E15, E19, E20, E21); enquanto que $42,9 \%$ pesquisaram o bullying $(n=9$; E4, E10, E11, E12, E13, E14, E16, E17, E18). Foi encontrado apenas 1 estudo que associou essas duas temáticas numa mesma pesquisa (4,7\%; E3). O compilado com as autorias, objetivos, populações e principais resultados de cada estudo encontra-se na Tabela 1.

Em relação às bases de dados, todos os estudos selecionados a partir da SciELO discutiram o abuso sexual ( $n=9$; E5, E6, E7, E8, E9, E15, E19, E20, E21). No sentido oposto, tanto a PsycINFO ( $n=4$; E11, E12, E13, E18) quanto a Scopus ( $n=4 ; E 4, E 10, E 16, E 17)$, trataram de abordar o bullying. Somente na PePSIC $(n=$ 4; abuso sexual: E1, E2; abuso sexual e bullying: E3; bullying: E14) foram encontrados estudos com ambos os fenômenos, isolados ou associados.

Destes, 42,85\% ( $n=9$; E1, E2, E3, E4, E5, E6, E7, E8, E9) das pesquisas foram realizadas no Brasil, 19,04\% ocorreram nos Estados Unidos ( $n=4$; E10, E11, E12, E13), 9,52\% em Portugal ( $n=2 ; E 14, E 15)$. Demais pesquisas foram realizadas na Espanha, Malásia, Reino Unido, Peru, Paraguai e México, correspondendo a 28,6\% dos estudos (respectivamente E16, E17, E18, E19, E20, E21). 
Figura 1. Etapa de seleção dos artigos incluídos na revisão integrativa sobre bullying e abuso sexual na adolescência

Número de artigos encontrados nas Bases de Dados $(n=3.787)$

Scielo $=79$, Scopus $=2.638$, PsycINFO $=1.056$ $\mathrm{PepSIC}=14$

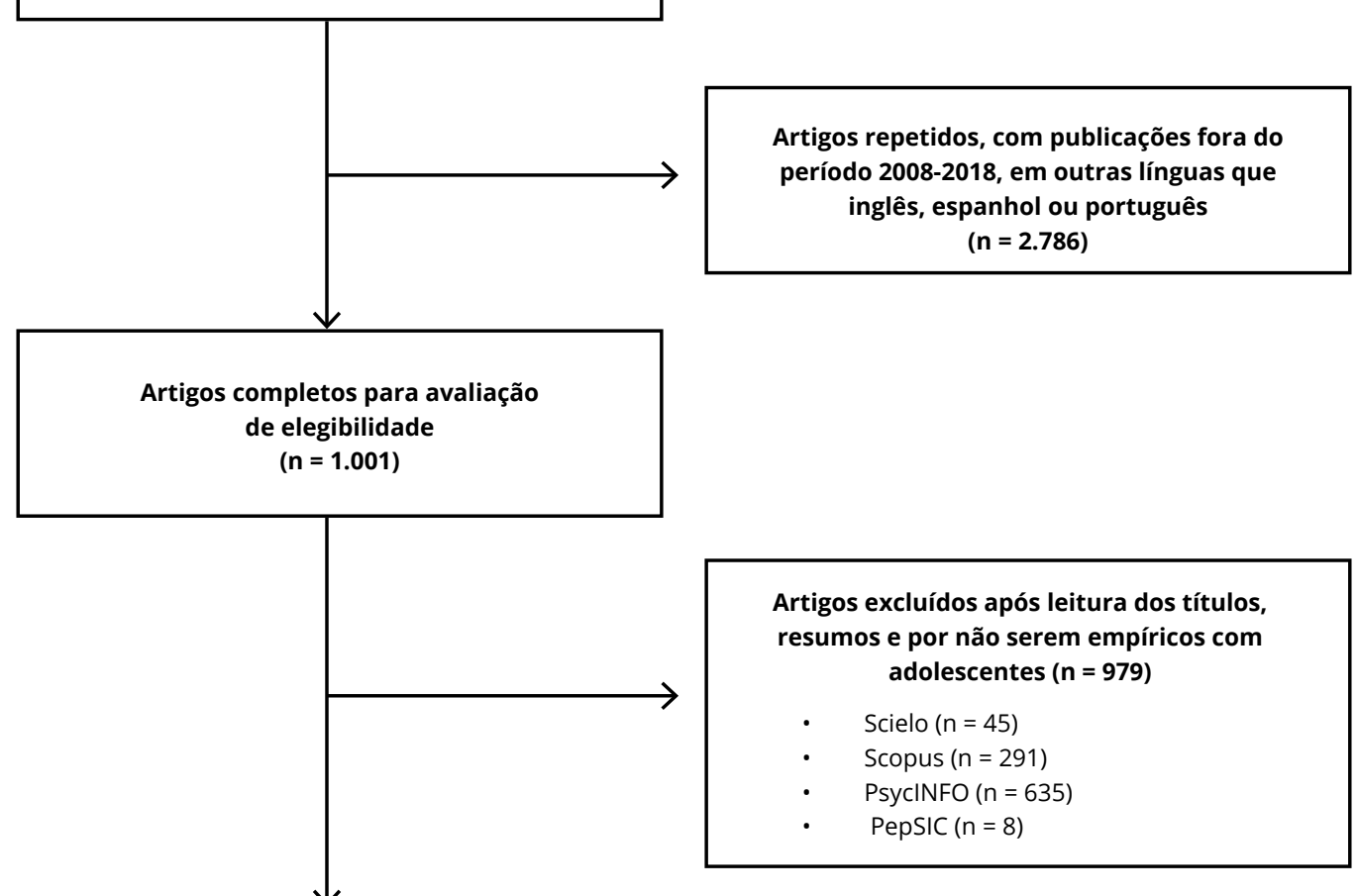

Estudos incluídos na síntese quantitativa $(\mathbf{n}=\mathbf{2 1})$

Scielo $=$ 9, Scopus $=4$, PsycINFO $=4$, PepSIC $=4$ 
Tabela 1. Síntese dos artigos que investigaram o bullying e/ou o abuso sexual em adolescentes (continua)

\begin{tabular}{|c|c|c|c|}
\hline Autoria, Ano, País, Área & Objetivo Principal & Participante e Instrumentos & Principais Resultados \\
\hline $\begin{array}{l}\text { (E1) Wolff et al., } 2016 \text { (Brasil; } \\
\text { Psicologia) }\end{array}$ & $\begin{array}{l}\text { Analisar a problemática } \\
\text { narcísica-identitária de } \\
\text { adolescentes que cometeram } \\
\text { abuso sexual. }\end{array}$ & $\begin{array}{l}4 \text { adolescentes do sexo masculino. } \\
\text { Entrevista e método de Rorschach. }\end{array}$ & $\begin{array}{l}\text { A dificuldade de definição dos limites, a problemática } \\
\text { identificatória, a inibição e o restrito manejo pulsional, bem } \\
\text { como o estabelecimento de relações especulares com o uso de } \\
\text { defesas narcísicas, indicam falhas no processo de subjetivação. }\end{array}$ \\
\hline $\begin{array}{l}\text { (E2) Silva \& Teixeira, } 2017 \text { (Brasil; } \\
\text { Psicologia) }\end{array}$ & $\begin{array}{l}\text { Conhecer, por meio da escuta } \\
\text { psicanalítica, as implicações } \\
\text { subjetivas referentes à vivência } \\
\text { de abuso sexual na } \\
\text { adolescência. }\end{array}$ & $\begin{array}{l}1 \text { adolescente do sexo feminino. Consultas } \\
\text { terapêuticas psicanalíticas. }\end{array}$ & $\begin{array}{l}\text { Além da cena de abuso sexual, há uma história de vida em um } \\
\text { contexto familiar específico, denotando marcas psíquicas que } \\
\text { antecedem a vivência do abuso, já que as primeiras relações } \\
\text { objetais e o investimento libidinal dispensado pela mãe- } \\
\text { ambiente são essenciais para a criança construir sua } \\
\text { identidade subjetiva e estabelecer relações objetais para o } \\
\text { longo da vida. }\end{array}$ \\
\hline $\begin{array}{l}\text { (E3) Miura et al., } 2018 \text { (Brasil; } \\
\text { Psicologia) }\end{array}$ & $\begin{array}{l}\text { Relatar as experiências das } \\
\text { autoras na condução de oficinas } \\
\text { realizadas com adolescentes em } \\
\text { uma escola de uma comunidade } \\
\text { litorânea de Maceió, Alagoas. }\end{array}$ & 26 adolescentes, ambos os sexos. Oficinas. & $\begin{array}{l}\text { A realização da proposta possibilitou o acesso a uma forma } \\
\text { diferente e potencializadora de vivenciar o ambiente escolar, } \\
\text { bem como aos temas (adolescência, gravidez na adolescência, } \\
\text { abuso sexual e bullying) que perpassam suas vivências } \\
\text { cotidianas. }\end{array}$ \\
\hline $\begin{array}{l}\text { (E4) Nascimento \& Menezes, } \\
2013 \text { (Brasil; Psicologia) }\end{array}$ & $\begin{array}{l}\text { Compreender os significados } \\
\text { produzidos sobre as práticas de } \\
\text { intimidação em contexto escolar. }\end{array}$ & $\begin{array}{l}28 \text { adolescentes, ambos os sexos. } \\
\text { Observação participante e debate focado } \\
\text { num tema para discussão. }\end{array}$ & $\begin{array}{l}\text { As interações que produziam intimidações, a partir dos valores } \\
\text { culturalmente significativos para o grupo, se sustentavam, pois } \\
\text { eram mascaradas pelo tom de brincadeira. }\end{array}$ \\
\hline $\begin{array}{l}\text { (E5) Borges \& Zingler, } 2013 \\
\text { (Brasil; Psicologia) }\end{array}$ & $\begin{array}{l}\text { Identificar os fatores de risco e } \\
\text { de proteção em casos de abuso } \\
\text { sexual na adolescência. }\end{array}$ & 2 adolescentes do sexo feminino. Entrevista. & $\begin{array}{l}\text { Percebeu-se uma presença maior de fatores de risco do que de } \\
\text { fatores de proteção no momento atual de vida das } \\
\text { adolescentes, o que merece uma atenção especial por parte } \\
\text { dos serviços que atendem estes casos. }\end{array}$ \\
\hline $\begin{array}{l}\text { (E6) Costa et al., } 2011 \text { (Brasil; } \\
\text { Psicologia) }\end{array}$ & $\begin{array}{l}\text { Comentar sobre o abuso sexual } \\
\text { praticado por esses } \\
\text { adolescentes desde dois pontos } \\
\text { principais: a adolescência vista } \\
\text { como fase de desenvolvimento e } \\
\text { o papel desempenhado pela } \\
\text { família na condução dessa fase }\end{array}$ & $\begin{array}{l}7 \text { adolescentes do sexo masculino. } \\
\text { Pesquisa-ação. }\end{array}$ & $\begin{array}{l}\text { Esses adolescentes revelaram carência de intimidade nas } \\
\text { relações parentais ocasionando a ausência de um espaço que } \\
\text { favorecesse a eles, de uma maneira segura, a dificuldade de } \\
\text { lidar com seus desejos. }\end{array}$ \\
\hline $\begin{array}{l}\text { (E7) Cantelmo et al., } 2011 \text { (Brasil; } \\
\text { Psicologia) }\end{array}$ & $\begin{array}{l}\text { Discutir a reorganização familiar } \\
\text { da adolescente, seu silêncio e o } \\
\text { de sua família em relação ao } \\
\text { abuso sexual. }\end{array}$ & $\begin{array}{l}1 \text { adolescentes do sexo feminino. } \\
\text { Observação participante. }\end{array}$ & $\begin{array}{l}\text { Traçou-se as Zonas de Sentido e indicadores de } \\
\text { vulnerabilidade: sua relação com a violência sofrida; sua } \\
\text { relaçãa com a família; sua relação com a filha e sua relação } \\
\text { com a escola. Nesse caso, o silêncio, o isolamento e a migração } \\
\text { para outra cidade foram opções de proteção. }\end{array}$ \\
\hline $\begin{array}{l}\text { (E8) Serafim et al., } 2011 \text { (Brasil; } \\
\text { Psicologia) }\end{array}$ & $\begin{array}{l}\text { Descrever os dados } \\
\text { demográficos e os aspectos } \\
\text { emocionais e comportamentais }\end{array}$ & $\begin{array}{l}205 \text { adolescentes, ambos os sexos. } \\
\text { Avaliação Psicológica e Psiquiátrica. }\end{array}$ & $\begin{array}{l}\text { Identificou-se uma parcela relevante de aspectos psicológicos, } \\
\text { psiquiátricos e comportamentais, os quais podem afetar de }\end{array}$ \\
\hline & $\begin{array}{l}\text { em crianças e adolescentes } \\
\text { vítimas de abuso sexual. }\end{array}$ & & $\begin{array}{l}\text { forma impactante o desenvolvimento emocional de crianças e } \\
\text { adolescentes. }\end{array}$ \\
\hline
\end{tabular}


Tabela 1. Síntese dos artigos que investigaram o bullying e/ou o abuso sexual em adolescentes (continuação)

\begin{tabular}{|c|c|c|c|}
\hline Autoria, Ano, País, Área & Objetivo Principal & Participante e Instrumentos & Principais Resultados \\
\hline $\begin{array}{l}\text { (E9) Habigzang et al., } 2008 \\
\text { (Brasil; Psicologia) }\end{array}$ & $\begin{array}{l}\text { Avaliar um modelo de } \\
\text { grupoterapia cognitivo- } \\
\text { comportamental para meninas } \\
\text { vítimas de abuso sexual. }\end{array}$ & $\begin{array}{l}10 \text { adolescentes do sexo feminino. } \\
\text { Entrevista semiestruturada; Escala de } \\
\text { atribuições e percepções de crianças; } \\
\text { Inventário de Depressão Infantil; Escala de } \\
\text { Estresse Infantil; Inventário de Ansiedade } \\
\text { Traço-Estado para crianças; Entrevista } \\
\text { estruturada baseada no DSM-IV. }\end{array}$ & $\begin{array}{l}\text { Pode-se concluir que foi efetiva e atuou na promoção de } \\
\text { resiliência e na melhoria da qualidade de vida das vítimas de } \\
\text { violência sexual e suas famílias. }\end{array}$ \\
\hline $\begin{array}{l}\text { (E10) Miller, } 2013 \text { (Estados } \\
\text { Unidos; Educação) }\end{array}$ & $\begin{array}{l}\text { Examinar o autoconceito de } \\
\text { sociabilidade dos alunos do } \\
\text { ensino médio e suas percepções } \\
\text { sobre se sentir seguro na escola. }\end{array}$ & $\begin{array}{l}420 \text { adolescentes, ambos os sexos. SSSQ: } \\
\text { Questionário de segurança e proteção } \\
\text { escolar; MTS: Teste Multidimensional de } \\
\text { Autoconceito. }\end{array}$ & $\begin{array}{l}\text { O autoconceito de sociabilidade amorteceu o efeito da } \\
\text { exposição à agressão na preocupação com a violência na } \\
\text { escola. }\end{array}$ \\
\hline $\begin{array}{l}\text { (E11) Evans \& Chapman, } 2014 \\
\text { (Estados Unidos; Psicologia) }\end{array}$ & $\begin{array}{l}\text { Examinar a experiências de } \\
\text { bullying em uma amostra racial } \\
\text { e etnicamente diversa de jovens } \\
\text { do ensino fundamental, médio e } \\
\text { médio rural. }\end{array}$ & $\begin{array}{l}3.379 \text { adolescentes, ambos os sexos. } \\
\text { Escala de Bullying. }\end{array}$ & $\begin{array}{l}\text { Jovens intimidados por serem chamados de gays, lésbicas ou } \\
\text { queer correm um alto risco de sofrer todas as formas de } \\
\text { comportamento de bullying, destacando a importância de um } \\
\text { maior apoio a esse grupo vulnerável. }\end{array}$ \\
\hline $\begin{array}{l}\text { (E12) Sharkey et al., } 2014 \\
\text { (Estados Unidos; Psicologia) }\end{array}$ & $\begin{array}{l}\text { Abordar a necessidade de } \\
\text { pesquisas sobre a associação } \\
\text { entre adotar ou negar o rótulo } \\
\text { de vítima de intimidação e o } \\
\text { funcionamento psicossocial dos } \\
\text { alunos. }\end{array}$ & $\begin{array}{l}1.063 \text { adolescentes, ambos os sexos. } \\
\text { Escala de vitimização do valentão da } \\
\text { Califórnia, Olweus Bully / Victim } \\
\text { Questionnaire, Questionário de pontos } \\
\text { fortes e dificuldades. }\end{array}$ & $\begin{array}{l}\text { A percepção de uma diferença de poder e o fato de terem sido } \\
\text { intimidados relacionam-se ao funcionamento psicossocial de } \\
\text { maneira interativa, sugerindo que ambos são importantes para } \\
\text { a consulta. Além disso, os estudantes que se rotularam como } \\
\text { vítimas de bullying relataram pior funcionamento psicossocial } \\
\text { do que aqueles que tiveram a experiência de sofrer bullying, } \\
\text { mas não adotaram esse rótulo. }\end{array}$ \\
\hline $\begin{array}{l}\text { (E13) Mitchell et al., } 2015 \\
\text { (Estados Unidos; Psicologia) }\end{array}$ & $\begin{array}{l}\text { Examinar as características e o } \\
\text { impacto emocional dos } \\
\text { incidentes de assédio entre } \\
\text { pares com base no grau de } \\
\text { envolvimento da tecnologia. }\end{array}$ & $\begin{array}{l}791 \text { adolescentes, ambos os sexos, } \\
\text { Entrevista via telefone. }\end{array}$ & $\begin{array}{l}\text { Os jovens que sofrem incidentes "mistos" de assédio entre } \\
\text { colegas devem ser uma prioridade para os educadores que } \\
\text { tentam identificar as experiências mais sérias e prejudiciais. }\end{array}$ \\
\hline $\begin{array}{l}\text { (E14) Lisboa et al., } 2014 \\
\text { (Portugal; Psicologia) }\end{array}$ & $\begin{array}{l}\text { Analisar a violência interpessoal } \\
\text { entre adolescentes (bullying) e } \\
\text { sua associação com a satisfação } \\
\text { com a vida e com a vida em } \\
\text { família. }\end{array}$ & $\begin{array}{l}\text { 4.954 adolescentes, ambos os sexos. } \\
\text { Questionário Comportamento em Saúde em } \\
\text { Crianças em Idade Escolar (HBSC). }\end{array}$ & $\begin{array}{l}\text { Equações de regressão múltipla indicaram que a satisfação } \\
\text { com a vida e com a família pode proteger os adolescentes de } \\
\text { se envolverem nesse tipo de violência } \\
\text { interpessoal (bullying). Diferenças de gênero sobre os } \\
\text { construtos estudados confirmaram estudos anteriores, e os } \\
\text { resultados permitem reflexão acerca da dinâmica de risco e } \\
\text { proteção que permeia as relações interpessoais dos } \\
\text { adolescentes, assim como sua interação com as características } \\
\text { da fase da adolescência. }\end{array}$ \\
\hline
\end{tabular}


Tabela 1. Síntese dos artigos que investigaram o bullying e/ou o abuso sexual em adolescentes (conclusão)

\begin{tabular}{|c|c|c|c|}
\hline Autoria, Ano, País, Área & Objetivo Principal & Participante e Instrumentos & Principais Resultados \\
\hline $\begin{array}{l}\text { (E15) Antunes \& Machado, } 2012 \\
\text { (Portugal; Psicologia) }\end{array}$ & $\begin{array}{l}\text { Compreender a experiência de } \\
\text { abuso e dos processos de } \\
\text { mudança associados. }\end{array}$ & $\begin{array}{l}16 \text { adolescentes, ambos os sexos. } \\
\text { Entrevista. }\end{array}$ & $\begin{array}{l}\text { Os recursos identificados pelos adolescentes para a mudança } \\
\text { incluem essencialmente a dimensão de suporte social (pares, } \\
\text { família e professores), sendo a revelação, as expectativas de } \\
\text { justiça, as crenças religiosas e a construção de novas } \\
\text { significações para o self, dimensões que reforçam a percepção } \\
\text { de competência pessoal e controle. }\end{array}$ \\
\hline $\begin{array}{l}\text { (E16) Schoeps et al., } 2018 \\
\text { (Espanha; Psicologia) }\end{array}$ & $\begin{array}{l}\text { Analisar o impacto de um } \\
\text { programa de educação } \\
\text { emocional em adolescentes para } \\
\text { promover a coexistência e o } \\
\text { bem-estar em sala de aula }\end{array}$ & $\begin{array}{l}148 \text { adolescentes, ambos os sexos, ESCQ } \\
\text { (Questionário de Habilidades e } \\
\text { Competências Emocionais); CYB-VIC - CYB- } \\
\text { AG (Escalas de cyberbullying); SWLS (Escala } \\
\text { de Satisfação com a Vida) }\end{array}$ & $\begin{array}{l}\text { A implementação de programas de intervenção em sala de } \\
\text { aula para desenvolver as competências emocionais dos alunos } \\
\text { pode ser benéfica para seu bem-estar subjetivo e coexistência } \\
\text { entre pares. }\end{array}$ \\
\hline $\begin{array}{l}\text { (E17) Bakar, } 2014 \text { (Malásia; } \\
\text { Tecnologia da Comunicação) }\end{array}$ & $\begin{array}{l}\text { Preencher a lacuna de } \\
\text { conhecimento no fenômeno do } \\
\text { cyberbullying, concentrando-se } \\
\text { em fatores que contribuem para } \\
\text { suas atividades entre os } \\
\text { adolescentes na Malásia. }\end{array}$ & $\begin{array}{l}105 \text { adolescentes, ambos os sexos. } \\
\text { Entrevista. }\end{array}$ & $\begin{array}{l}\text { O cyberbullying produz vários impactos prejudiciais aos } \\
\text { adolescentes. Os entrevistados relataram equivalentemente } \\
\text { níveis mais altos de incidentes de cyberbullying quando eles se } \\
\text { registraram no Facebook. }\end{array}$ \\
\hline $\begin{array}{l}\text { (E18) Ferguson \& Colwell, } 2016 \\
\text { (Reino Unido; Psicologia) }\end{array}$ & $\begin{array}{l}\text { Examinar as relações } \\
\text { correlacionais entre exposição } \\
\text { violenta ao jogo e } \\
\text { comportamentos de bullying, } \\
\text { atitudes antissociais, atitudes } \\
\text { cívicas e comportamentos } \\
\text { cívicos. }\end{array}$ & $\begin{array}{l}304 \text { adolescentes, ambos os sexos. } \\
\text { Placa de classificação de software de } \\
\text { entretenimento, Escala de eventos de vida } \\
\text { negativos, Escala de envolvimento parental, } \\
\text { Escala de Bullying de Olweus e Escala de } \\
\text { atitudes cívicas e comportamentos cívicos. }\end{array}$ & $\begin{array}{l}\text { A exposição violenta ao jogo não se correlacionou } \\
\text { significativamente com comportamentos ou atitudes } \\
\text { antissociais ou cívicos. Esses resultados são discutidos em um } \\
\text { contexto motivacional e de desenvolvimento. }\end{array}$ \\
\hline $\begin{array}{l}\text { (E19) Moyano et al., } 2016 \text { (Peru; } \\
\text { Psicologia) }\end{array}$ & $\begin{array}{l}\text { Explorar a relação entre o duplo } \\
\text { padrão sexual e as atitudes de } \\
\text { apoio ao estupro em relação à } \\
\text { probabilidade de um indivíduo } \\
\text { de perpetrar agressões sexuais. }\end{array}$ & $\begin{array}{l}448 \text { adolescentes do sexo masculino. } \\
\text { Questionário sociodemográfico; Escala } \\
\text { sobre o duplo padrão sexual; Escala de } \\
\text { atitudes de apoio ao estupro; Pesquisa } \\
\text { sobre experiências sexuais. }\end{array}$ & $\begin{array}{l}\text { Os achados sugerem que atitudes violentas são mais } \\
\text { importantes do que o endosso de crenças não igualitárias } \\
\text { (duplo padrão sexual) na perpetração da violência sexual. }\end{array}$ \\
\hline $\begin{array}{l}\text { (E20) Arrom et al., } 2015 \\
\text { (Paraguai; Ciências da Saúde) }\end{array}$ & $\begin{array}{l}\text { Determinar a frequência de } \\
\text { abuso sexual e outras formas de } \\
\text { violência em estudantes de nível } \\
\text { médio de colégios do Alto } \\
\text { Paraná. }\end{array}$ & $\begin{array}{l}661 \text { adolescentes, ambos os sexos. } \\
\text { Questionário. }\end{array}$ & $\begin{array}{l}\text { A tentativa de abuso sexual ou abuso em algum momento, } \\
\text { muitos deles dentro do círculo familiar. A crescente gravidade } \\
\text { do problema destaca a vulnerabilidade desse grupo } \\
\text { populacional, o que sugere a necessidade de implementar } \\
\text { programas para prevenir a violência e principalmente o abuso } \\
\text { sexual em crianças e adolescentes. }\end{array}$ \\
\hline $\begin{array}{l}\text { (E21) Ayala et al., } 2009 \text { (México; } \\
\text { Ciências da Saúde) }\end{array}$ & $\begin{array}{l}\text { Estimar a prevalência e os } \\
\text { fatores associados ao abuso } \\
\text { sexual na infância e } \\
\text { adolescência. }\end{array}$ & $\begin{array}{l}1.730 \text { adolescentes, ambos os sexos. } \\
\text { Questionário. }\end{array}$ & $\begin{array}{l}\text { Em média, o abuso sexual ocorre aos } 12 \text { anos de idade em } \\
\text { ambos os sexos, sendo mais frequente no sexo feminino. A } \\
\text { maioria das vítimas não denuncia. }\end{array}$ \\
\hline
\end{tabular}


Quanto a área de concentração dos periódicos, $80,95 \%$ dos estudos pertenciam à psicologia $(n=$ 17; E1, E2, E3, E4, E5, E6, E7, E8, E9, E11, E12, E13, E14, E15, E16, E18, E19), e 2 estudos foram desenvolvidos por pesquisadores ligados às ciências da saúde (9,52\%; E20, E21). Demais pesquisas estavam ligadas à educação (E10) e tecnologia da comunicação (E17), correspondendo a 9,52\% dos estudos. No que diz respeito ao ano de publicação, houve maior concentração de publicações nos últimos cinco anos $(57,15 \% ; n=12 ; E 1, E 2, E 3, E 11, E 12, E 13, E 14, E 16$, E17, E18, E19, E20) se comparado aos cinco anos anteriores a esse período $(42,85 \%, n=9$; E4, E5, E6, E7, E8, E9, E10, E15, E21).

\section{Características amostrais, tipo de estudo e instrumentos utilizados}

Os estudos totalizaram 14.303 participantes, sendo que em relação a menor amostra, 2 estudos foram compostos por apenas 1 indivíduo (E2, E7) e a maior totalizou 4.954 pessoas (E14). Os dois primeiros estudos foram realizados nos Brasil e objetivaram: conhecer, por meio da escuta psicanalítica, as implicações subjetivas referentes à vivência de abuso sexual na adolescência; discutir a reorganização familiar da adolescente, seu silêncio e o de sua família em relação ao abuso sexual (respectivamente). Já o segundo foi realizado em Portugal e buscou analisar a violência interpessoal entre adolescentes (bullying) e sua associação com a satisfação com a vida e com a vida em família.

As idades dos participantes variaram entre 6 (E8) a 24 anos (E21), e composição predominante de sexo misto em $61,9 \%$ dos estudos ( $n=14$; E3, E4, E8, E10, E11, E12, E13, E14, E15, E16, E17, E18, E20, E21). Enquanto que $19,04 \%$ das pesquisas foram realizadas apenas com participantes do sexo feminino ( $n=4$; E2, E5, E7, E9) e $14,3 \%$ masculino ( $n=3$; E1, E6, E19).

Em 52,4\% dos artigos selecionados ( $n=11 ; E 3, E 4$, E10, E11, E12, E14, E16, E17, E18, E20, E21), os participantes foram recrutados em escolas. Enquanto que nos demais estudos, o contato com os adolescentes em ambientes não-escolares. A investigação acerca dos dados sociodemográficos (situação financeira da família, escolaridade, etnia, religião, etc.) esteve ausente em $71,42 \%$ dos estudos $(n=15 ; E 1, E 2$, E3, E4, E5, E9, E12, E13, E10, E14, E15, E16, E17, E18, E19). Nos demais artigos essas variáveis não foram incluídas totalmente em suas análises, não havendo uma caracterização completa das amostras nesses aspectos.

Quanto ao desenho de pesquisa, percebeu-se uma variedade de modelos metodológicos. 38,09\% dos estudos eram quantitativos com a utilização de escalas e/ou questionários ( $\mathrm{n}=8$; E10, E11, E12, E13, E14, E18, E19, E21), ainda sob esse aspecto, convém ressaltar que o E13 foi realizado via telefone. Estudos qualitativos corresponderam a $57,15 \%$ dos artigos ( $n=12 ; E 1$, E2, E3, E4, E5, E6, E7, E8, E9, E15, E17, E20). Apenas 1 estudo (4,76\%; E16) utilizou metodologia quase-experimental, sendo que os demais foram do tipo não experimental.

A maioria dos estudos utilizou um instrumento (ou mais) para coletar dados e atingir seus objetivos. Destes, 33,33\% utilizaram escalas e, em alguns casos, questionários também ( $n=7$; E9, E10, E11, E12, E16, E18, E19). Não foi percebida a utilização de um mesmo instrumento em dois ou mais estudos. Algumas escalas eram até da mesma autoria, mas com propósitos diferenciados (E12, E18). Os estudos que só foram realizados com a utilização de questionários corresponderam a 14,28\% do total ( $n=3 ; E 14, E 20, E 21)$.

\section{Características dos estudos que abordaram o bullying}

Dos estudos voltados a pesquisar e discutir o bullying, apenas o E13 não foi realizado dentro do contexto escolar, em razão das entrevistas terem sido realizadas via telefone. Dois estudos não direcionaram o bullying a um ou mais fenômenos específicos: E4 e E17. O objetivo do E4 foi compreender os significados produzidos sobre as práticas do bullying escolar; enquanto que no E17, apesar da semelhança no objetivo, a principal diferença é que o bullying em questão foi o cibernético. Em contrapartida, nos demais estudos, o bullying foi pesquisado em relação direta com: autoconceito de sociabilidade e percepção de segurança (E4); amostra racial e etnicamente diversa (E11); rótulos (E12); impacto emocional (E13); satisfação de vida e com a vida em família (E14); programa de educação emocional (E16); exposição a jogos violentos (E18).

Com relação ao cerne dos principais resultados, cada estudo destacou-se por sua singularidade. No E4 ficou evidente que a problemática do bullying nas escolas, muitas das vezes, é mascarada por ser considerada como meras brincadeiras; enquanto que através do 
E10, verificou-se que o autoconceito de sociabilidade atenua os efeitos do bullying e a preocupação com a violência escolar. O E11 considerou que gays, lésbicas ou queer estão mais propensos a sofrerem bullying; e o E12 afirmou que entre os alvos de bullying aqueles que aderiram aos rótulos tiveram pior funcionamento psicossocial.

O E13 asseverou que o atendimento deve ser priorizado para aqueles alvos de bullying que mais sofreram impactos emocionais. Já o E14 trouxe em suas considerações que pessoas com maior índice de satisfação de vida em família são mais aptas a se protegerem de bullying. No tocante ao E16, ressaltou-se que a implantação de programa de educação emocional é benéfica para o enfrentamento do bullying. O E17 apontou que no contexto do cyberbullying na Malásia, o grande provocador de impactos negativos é o Facebook. E por fim, o E18 considerou que a exposição a jogos violentos não teve relação direta com comportamentos de bullying, sendo atribuídos, então, o contexto motivacional e de desenvolvimento.

\section{Características dos estudos que abordaram o abuso sexual}

Nos estudos sobre o abuso sexual percebeu-se três direcionamentos principais tanto no que diz respeito aos participantes, quanto aos propósitos das pesquisas. O primeiro esteve voltado a colher relatos baseados nas vivências dos adolescentes cujos pesquisadores já sabiam que, em algum momento, foram vítimas de abuso sexual ( $n=6$; E2, E5, E7, E8, E9, E15). A coleta do segundo foi direcionada para os adolescentes que, em algum momento, perpetraram o abuso sexual ( $n=2 ; E 1, E 6)$. No terceiro, por sua vez, o foco da coleta foram adolescentes que nem sofreram e nem praticaram o abuso sexual, no intuito de que eles expressassem seus entendimentos acerca do fenômeno ( $n=2 ; E 20, E 21)$. Vale ressaltar que o E19 fugiu a essa regra, pois sua pesquisa foi composta por 448 adolescentes do sexo masculino, dos quais 148 (33.3\%) informaram já terem perpetrado o abuso sexual. Em outras palavras, o propósito foi fazer o comparativo de entendimentos sobre a matéria, entre quem já praticou e quem nunca se envolveu com violência sexual.

Os estudos mostraram que a grande maioria das tentativas e o próprio abuso sexual acontecem dentro do círculo familiar (E20). Que a idade média das vítimas foi no entorno de12 anos (E21), que o sexo das vítimas mais prevalente é o feminino (E2, E5, E7, $E 9, E 21)$, mas que também acontece com pessoas do sexo masculino (E15, E8). Quanto aos perpetradores, o sexo masculino mostrou-se ser o mais frequente (E1, E6, E19). E neste sentido, o E6 afirmou que a carência de intimidade nas relações parentais é o campo fértil para o adolescente ampliar a dificuldade de lidar com seus desejos e tornar mais propenso a perpetração de violências sexuais.

Quanto aos fatores que contribuem para que esse fenômeno seja frequente entre os adolescentes, os estudos apontaram a subnotificação dos casos (E21) e uma presença maior de fatores de risco do que de fatores de proteção (E5).

Características do estudo que associou o bullying e o abuso sexual na mesma pesquisa

O E3 tratou-se de apresentar as experiências provenientes de oficinas realizadas com adolescentes numa escola de Maceió. Dentre as oficinas foram abordados o bullying e o abuso sexual, assim como também adolescência e gravidez. Percebeu-se que os alunos detinham, minimamente, de conhecimentos acerca destes fenômenos, que além das reflexões em grupo, se propuseram a pôr em práticas medidas de enfrentamento.

\section{Discussão}

A revisão integrativa é considerada a mais ampla abordagem metodológica no que diz respeito às revisões, pois permite a inclusão de estudos experimentais e não-experimentais para uma compreensão mais ampla do fenômeno analisado (Souza, Silva \& Carvalho, 2010). Com essa finalidade, o objetivo do presente estudo foi reunir as principais discussões associando adolescentes e situações de bullying e/ou abuso sexual, publicadas na literatura científica.

Observou-se que, quanto ao número de publicações selecionadas, por diferença de dois estudos a mais, o abuso sexual se sobressaiu (Antunes \& Machado, 2012; Arrom et al., 2015; Ayala et al., 2009; Borges \& Zingler, 2013; Cantelmo et al., 2011; Costa et al., 2011; Habigzang et al., 2008; Moyano et al., 2016; Serafim et al., 2011; Silva \& Teixeira, 2017; Wolff et al., 2016). Este dado entra em consonância com a realidade acadêmica atual, pois tem-se percebido grandes 
investimentos na construção do conhecimento científico a partir da investigação do abuso sexual em crianças e adolescentes. Esse movimento tem gerado um acúmulo de conhecimento que vem favorecendo a sociedade por meio da construção de políticas públicas dedicadas à intervenção e tratamento das vítimas (Marra \& Costa, 2018). Seguindo esse mesmo sentido, o bullying entre adolescentes também é um tema largamente pesquisado tanto no Brasil quanto em várias partes do mundo (Crochík, 2016; Ficher, 2015; Rajput et al., 2018).

O fato de ter sido selecionado apenas um estudo (Miura et al., 2018) que associou o bullying e o abuso sexual numa mesma pesquisa com adolescentes demonstra carência de investigações científicas com este fim. Haja vista que o abuso sexual (nas variações: abuso sexual verbal, assédio sexual, exibicionismo, voyeurismo, estupro, corrupção e atentado violento ao pudor, etc.) pode ser perpetrado na forma de bullying do tipo verbal ou com contato físico (Antunes \& Zuin, 2008; Landini, 2011).

Reforçando esse entendimento, pesquisa realizada com 1.162 estudantes dos Estados Unidos revelou que os garotos (37\%) são mais autores de bullying que as garotas (28\%), no tocante às questões que envolvem a sexualidade. Enquanto alvos, o bullying verbal de cunho sexual foi o mais frequente em $73 \%$ das garotas versus $66 \%$ dos garotos. Os adolescentes relataram, ainda, que a violência sexual física na forma de bullying também acontece, apesar de ser menos comentada (Espelage et al., 2014).

No que diz respeito aos países onde foram realizadas as pesquisas, houve variedade referente à distribuição (Brasil, Estados Unidos, Portugal, Reino Unido, Espanha, Malásia, Peru, Paraguai e México). Dados atuais já confirmaram que tanto o bullying quanto o abuso sexual são consideradas questões de saúde pública não só no Brasil, mas também em todo o mundo (Oliveira et. al., 2017; Platt et al., 2018). No contexto do Brasil, levantamento realizado através do dispositivo de denúncias "Disque 100", revelou que, no período de 2003 a 2010, das mais de 145.000 denúncias, as violências físicas e psicológicas, das quais destaca-se o bullying, empatou em porcentagem com a "negligência" (ambas com 34\%, cada) seguida das violências sexuais (32\%) (Brasil, 2018).

Concernente à área de concentração dos estudos selecionados, a maioria foi publicada em periódicos da psicologia (80,95\%). Esse fato mostrou que o campo de pesquisa e prática é vasto e vem sendo explorado, primordialmente, pela psicologia, assim como em outras áreas, a saber, ciências da saúde, educação, tecnologia da informação). Diante disso, compreende-se o quão tem sido relevantes as contribuições a partir do aporte teórico-técnico da psicologia, que é marcada por uma grande diversidade de teorias, perspectivas profissionais, objetos de análise, epistemologias, ontologias, metodologias de pesquisa e/ou intervenção, temas e enfoques (Machado, 2011).

A razão para que a maioria dos estudos serem realizados em escolas, possivelmente, seja em função, além da conveniência de ser o ambiente propício para se encontrar um grande número de adolescentes reunidos, como também por ser o espaço adequado para a promoção de discussões relevantes para sociedade, visto que uma das principais finalidades da escola é preparar o jovem para o convívio social (Oliveira et al., 2013).

No tocante ao desenho das pesquisas, percebeu-se maior número de estudos com abordagens qualitativas $(57,15 \%)$, pois, dessa forma, foi possível se ocupar do nível subjetivo e relacional da realidade social, por meio da história, do universo, dos significados, dos motivos, das crenças, dos valores e das atitudes (Minayo, 2013) dos adolescentes que vivenciaram situações de bullying e/ou abuso sexual. Mas, em contrapartida, os estudos com abordagem quantitativa também se destacaram (38,09\%), da qual recorreu à linguagem matemática para descrever as causas dos fenômenos, as relações entre variáveis, etc. (Fonseca, 2002), pautados na busca por objetividade no que se refere à definição de variáveis, de hipóteses e, principalmente, no rigor da mensuração das variáveis e criação de modelos. Com isso, tais características, supostamente, oferecem garantia em maior escala de segurança, ou até mesmo a minimização das distorções nas interpretações dos resultados (Nobre et al., 2017).

Dos estudos que não direcionaram o bullying a um ou mais fenômenos específicos (Bakar, 2014; Nascimento \& Menezes, 2013), percebeu-se neles o intuito de descrever sua ocorrência, tal como foi concebido pelos adolescentes no ambiente escolar e por meio das mídias sociais digitais. Esse tipo de pesquisa ratifica a importância de estudar os fatores de risco/ proteção associados ao bem-estar subjetivo, desde a percepção que adolescentes têm acerca do bullying e 
dos âmbitos de desenvolvimento, seja na escola, casa ou nas redes sociais (Alcântara et al., 2019).

Nos demais estudos (Evans \& Chapman, 2014; Ferguson \& Colwell, 2016; Lisboa et al., 2014; Miller, 2013; Mitchell et al., 2015; Schoeps et al., 2018; Sharkey et al., 2014), o bullying foi pesquisado em relação direta a um ou mais fenômenos, demonstrando que não foram esgotadas todas as possibilidades de sua manifestação, principalmente, por não possuir alvos específicos, pré-estabelecidos, a não ser a fragilidade percebida. Convém ressaltar que, para alguns autores, na base dessa dinâmica está o preconceito, pois esses alvos são pré-julgados e justificados para o exercício de violência que sofrem. A própria conceituação de bullying se aproxima do conceito de preconceito, em especial, quando se propõe a refletir sobre os fatores sociais que determinam os grupos-alvo, bem como sobre o funcionamento psíquico entre os considerados agressores (Antunes \& Zuin, 2008; Crochík, 2019; Jodelet, 2006).

Ainda quanto à diversidade de abordagens de estudos sobre o bullying, a literatura científica demonstra que as pesquisas no âmbito internacional, na sua grande maioria, investigam participantes de ambos os sexos (Fox \& Boulton, 2005). Demonstrando o quão é relevante ampliar cada vez mais as possibilidades de pesquisas, independentemente do gênero ou grupo etário envolvidos neste tipo de violência, muitas das vezes disfarçada de brincadeiras entre pares. Uma das razões, para tanto, está no desencadeamento de consequências, que vão desde uma angústia até o assassinato e suicídio (Antunes \& Zuin, 2008; Fante, 2005).

Em relação aos três direcionamentos na seleção de participantes nas pesquisas sobre o abuso sexual, observou-se que a comunidade científica não limitou o seu olhar somente para um foco, mas levou em consideração as vicissitudes que compreendem este fenômeno, como também nos estudos sobre o bullying. Em outras palavras, no contexto do abuso sexual, foram coletadas informações não só das vítimas, mas também dos perpetradores e de adolescentes sem relação direta com o abuso sexual. Assim como afirmam Martins e Jorge (2010), implementar estudos científicos com essas conformações é essencial para conhecer melhor o evento, incluindo sua relação com os fatores predisponentes, 0 que pode direcionar melhor as ações de enfrentamento.
Enquanto que no contexto do bullying, as diversas relações com outros fenômenos reforçaram o entendimento que se trata de algo complexo e que se faz necessário ainda mais investimentos em pesquisas, para que seja possível compreender suas diversas facetas (Zequinão et al., 2016).

Conforme enfatizado nos estudos selecionados, no tocante ao local onde o abuso sexual é mais perpetrado, pesquisa realizada com 9 famílias atendidas por um Centro de Referência Especializado de Assistência Social (CREAS) relevou que o abuso sexual intrafamiliar prevalece sobre o extrafamiliar, e que a maioria das vítimas é de meninas (Marra \& Costa, 2018). Também entraram em consonância com a literatura científica ao afirmarem que a maioria dos perpetradores é do sexo masculino, pessoas de confiança da vítima, e que o abuso, normalmente, ocorre na casa da vítima ou na circunvizinhança (Landini, 2011; Martins \& Jorge, 2010).

Estudos nacionais e internacionais apontam que o sexo feminino é a vítima mais prevalente de abuso sexual (Borges \& Zingler, 2013; Cantelmo et al., 2011; Habigzang et al., 2008; OMS, 2003; Santos et al., 2018; Silva \& Teixeira, 2017); mas, também revelam que, mesmo sendo em menor proporção, em torno de $5 \%$ dos meninos até completarem a maioridade, em algum momento, foram vítimas de abuso sexual com penetração, sem levar em consideração as outras formas de abuso (Said \& Costa, 2019).

Quanto a idade das vítimas, não há consenso em relação a uma faixa etária precisa. Estudos recentes apontam dados que variaram desde em torno dos 7 anos (Mascarenhas et al., 2018), 8 anos (Marra \& Costa, 2018), e 10 anos para meninas, 9 anos para meninos (Martins \& Jorge, 2010). Entretanto, a faixa etária em torno dos 12 anos é mais disseminada na literatura científica (Landini, 2011), conforme afirmaram Ayala et al. (2009).

Este mesmo estudo (Ayala et al., 2009) também chamou atenção para o problema da subnotificação dos casos, o qual se configura um problema grave. Pois é por meio de dados epidemiológicos que o Estado implementa políticas públicas, que vão desde a educação em saúde, prevenção, enfrentamento e punição. A efetuação das denúncias pode diminuir (e até mesmo acabar) com outra forma de violência no nível estrutural: a invisibilidade, que é desencadeadora de descaso (Azambuja, 2005). 
Ao tratar-se de adolescentes de modo geral e, principalmente, quando envolvidos em situações de violências (neste caso, bullying e abuso sexual), torna-se prudente discutir os fatores de risco e de proteção. No contexto do estudo de Borges e Zingler (2013) foi verificado que os fatores de proteção foram mais escassos em detrimento dos fatores de risco. Tais fatores não podem ser analisados de forma mecânica, descontextualizados, a-históricos e individualizados, porque é por meio deles que se obtém melhor compreensão de vários elementos que constituem a realidade dos adolescentes (Euzébios Filho \& Guzo, 2006).

Como se percebeu por meio desta revisão integrativa, embora existam inúmeras pesquisas que abordem as características da ocorrência da violência sexual contra adolescentes no Brasil e no mundo (Santos et al., 2018), ainda há poucos registros na literatura que $o$ associe ao bullying em pesquisas realizadas no ambiente escolar, conforme relatado por Miura et al., 2018. Haja vista que ambos são desencadeadores de consequências nocivas (Chaves \& Sousa, 2018; Fante, 2005; Florentino, 2015; Landini, 2011; Molcho et al., 2009; Olweus, 1993; Pereira, 2008), e que o abuso sexual pode ser perpetrado como uma das manifestações do bullying (Antunes \& Zuin, 2008; Landini, 2011).

\section{Considerações Finais}

Esta revisão integrativa buscou reunir os principais estudos sobre o bullying e o abuso sexual, como violências em que os adolescentes estão susceptíveis diuturnamente. Além das características e objetivos, foram observados também as metodologias e subtemas, assim como, por meio dos resultados, vislumbrou-se quais foram os principais achados e discussões nas publicações entre 2008-2018.

Em nível quantitativo, o abuso sexual foi a violência que mais se sobressaiu dentre os estudos selecionados. Este dado ratificou a importância da constante discussão dessa temática, pois tanto a sexualidade quanto o abuso sexual, em alguns contextos, ainda são considerados assuntos tabus (Inoue \& Ristum, 2008). O bullying, por sua vez, também se destacou nessa revisão. Trata-se, portanto, de um assunto em evidência na sociedade e na comunidade científica, pois diferentemente do abuso sexual que, por vezes, pode ser velado, o bullying é mais fácil ser vislumbrado, principalmente, nos contextos das escolas (Pigozi \& Machado, 2015).

Foram limitações nessa revisão integrativa a restrição aos estudos empíricos de campo e o restringimento de participantes adolescentes, que não impossibilitaram o alcance do objetivo proposto. Por ser selecionado apenas 1 estudo que associou ambos os fenômenos, sugere-se, portanto, que novas investigações desse tipo sejam implementadas, bem como analisando o abuso sexual como manifestação de bullying.

\section{Contribuições dos autores}

Felizmino, T. O. atuou desde o planejamento da pesquisa, na coleta dos dados, bem como nas análises e na redação do artigo. Silva, J. P. orientou o trabalho, apoiou no planejamento do estudo, orientou a coleta dos dados, bem como apoiou na análise e redação do artigo.

\section{Conflitos de interesses}

Nenhum conflito financeiro, legal ou político envolvendo terceiros (governo, empresas e fundações privadas, etc.) foi declarado para nenhum aspecto do trabalho submetido (incluindo, mas não se limitando a subvenções e financiamentos, participação em conselho consultivo, desenho de estudo, preparação de manuscrito, análise estatística, etc.).

\section{Referências}

Alcantara, S. C., González-Carrasco, M., Montserrat, C., Casas, F., Viñas-Poch, F., \& Abreu, D. P. (2019). Violência entre pares, clima escolar e contextos de desenvolvimento: suas implicações no bem-estar. Ciência \& Saúde Coletiva, 24(2), 509-522. https://doi.org/10.1590/1413$\underline{81232018242.01302017}$

Antunes, J., \& Machado, C. (2012). Violência nas relações íntimas ocasionais de uma amostra estudantil. Análise Psicológica, 30(1-2), 93-107. http://publicacoes.ispa.pt/index.php/ap/ article/view/535

Antunes, D.C., \& Zuin, A. A. (2008). Do bullying ao preconceito: os desafios da barbárie à educação. Psicologia \& Sociedade, 20(1), 33-41. https://doi.org/10.1590/5010271822008000100004

Arrom, C. H., Ruoti, M., Orué, E., \& Arrom, C. M. (2015). Abuso sexual y otras formas de violencia en estudiantes de nivel medio en el departamento de alto Paraná [Abuso sexual e outras formas de violência em estudantes do ensino 
médio do departamento do Alto Paraná]. Memorias del Instituto de Investigaciones en Ciencias de la Salud, 13(3), 31-38. https://pesquisa.bvsalud.org/portal/resource/pt/ biblio-869062

Associação Americana de Psicologia. (2010). Dicionário de psicologia da APA. Artmed.

Ayala, R. C., Rivera-Rivera, L., Angeles-Llerenas, A., Díaz-Cerón, E., Allen-Leigh, B., \& Ponce, E. L. (2009). Factores del abuso sexual en la niñez y la adolescencia en estudiantes de Morelos, México [Fatores de abuso sexual na infância e adolescência de estudantes de Morelos, México]. Revista de Saúde Pública, 43(3), 506-514. https://www.scielo.br/j/ rsp/a/jCFLtcndmNDYb8SJdf6HSkr/?lang=es

Azambuja, M. P. R. (2005). Violência doméstica: reflexões sobre o agir profissional. Psicologia: Ciência e Profissão, 25(1), 4-13. https://doi.org/10.1590/S1414-98932005000100002

Bakar, H. S. A. (2015). The emergence themes of cyberbullying among adolescences [O surgimento do tema de cyberbullying entre adolescentes]. International Journal of Adolescence and Youth, 20(4), 393-406. https://doi.org/10.10 $\underline{80 / 02673843.2014 .992027}$

Borges, J. L., \& Zingler, V. T. (2013). Fatores de risco e de proteção em adolescentes vítimas de abuso sexual. Psicologia em Estudo, 18(3), 453-463. https://www.scielo.br/j/pe/a/ sVss7wWTqkQGkTnH8PPgLbN/abstract/?lang=pt

Braga, L. L., \& Dell'Aglio, D. D. (2012). Exposição à violência em adolescentes de diferentes contextos: família e instituições. Estudos de Psicologia, 17(3), 413-420. https:// doi.org/10.1590/S1413-294X2012000300009

Ministério dos Direitos Humanos. (2018). Violência contra Crianças e Adolescentes: Análise de Cenários e Propostas de Políticas Públicas. Ministério dos Direitos Humanos. https://www. gov.br/mdh/pt-br/centrais-de-conteudo/consultorias/ conada/violencia-contra-criancas-e-adolescentes-analisede-cenarios-e-propostas-de-politicas-publicas.pdf

Cantelmo, C. A., Cavalcante, T. P., \& Costa, L. F. (2011). A menina mãe: incesto e maternidade. Fractal: Revista de Psicologia, 23(1), 137-154. https://doi.org/10.1590/S198402922011000100010

Chaves, D. R. L., \& Souza, M. R. (2018). Bullying e preconceito: a atualidade da barbárie. Revista Brasileira de Educação, 23, e230019. https://doi.org/10.1590/S1413-24782018230019

Costa, L. F., Junqueira, E. L., Ribeiro, A., \& Meneses, F. F. F. (2011). "Ministério da Obrigação adverte": é preciso proteger os adolescentes ofensores sexuais. Avances em Psicologia Latinoamericana, 29(1), 33-46. https://www.redalyc.org/ articulo.oa?id=79920065004

Crochík, J. L. (2015). Formas de violência escolar preconceito e bullying. Movimento-Revista de Educação, 3. https://doi. org/10.22409/mov.v0i3.270
Crochík, J. L. (2016). Educação Inclusiva: algumas pesquisas. Novas Edições Acadêmicas.

Crochík, J. L. (2019). Preconceito e bullying: marcas da regressão psíquica socialmente induzida. Psicologia USP, 30, e190006. https://doi.org/10.1590/0103-6564e190006

Espelage, D. L., Low, S. K., Anderson, C., \& De la Rue, L. (2014). Bullying, sexual, and dating violence trajectories from early to late adolescence [Trajetórias de bullying, violência sexual e namoro desde o início até o final da adolescência]. Methodology, 9(11), 1-74. https://www.ojp. gov/pdffiles1/nij/grants/246830.pdf

Euzébios Filho, A., \& Guzzo, R. S. L. (2006). Fatores de risco e de proteção: percepção de crianças e adolescentes. Temas em Psicologia, 14(2), 125-141. http://pepsic.bvsalud.org/pdf/tp/ v14n2/v14n2a03.pdf

Evans, C. R., \& Chapman, M. V. (2014). Bullied youth: the impact of bullying through lesbian, gay, and bisexual name calling [Juventude intimidada: o impacto do bullying através do nome de lésbicas, gays e bissexuais]. American Journal of Orthopsychiatry, 84(6), 644-652. https://doi.org/10.1037/ ort0000031

Facuri, C. D. O., Fernandes, A. M. D. S., Oliveira, K. D., Andrade, T. D. S., \& Azevedo, R. C. S. D. (2013). Violência sexual: estudo descritivo sobre as vítimas e o atendimento em um serviço universitário de referência no Estado de São Paulo, Brasil. Caderno de Saúde pública, 29(5), 889-898. https:// doi.org/10.1590/S0102-311X2013000500008

Fante, C. (2005). Fenômeno bullying: como prevenir a violência nas escolas e educar para a paz. Versus.

Ferguson, C. J., \& Colwell, J. (2016). A meaner, more callous digital world for youth? The relationship between violent digital games, motivation, bullying, and civic behavior among children [Um mundo digital mais cruel e insensível para a juventude? A relação entre jogos digitais violentos, motivação, intimidação e comportamento cívico entre as crianças]. Psychol Pop Med Cult, 7(3), 202. https://psycnet. apa.org/doi/10.1037/ppm0000128

Fisher, C. (2015). The association of different types of bullying with the mental health of children and teens from the United States, France, and Canada [A associação de diferentes tipos de bullying com a saúde mental de crianças e adolescentes dos Estados Unidos, França e Canadá]. Media and Communication Studies Summer Fellows, 4. https://digitalcommons.ursinus.edu/cgi/viewcontent. cgi?article $=1003 \&$ context $=$ media_com_sum

Florentino, B. R. B. (2015). As possíveis consequências do abuso sexual praticado contra crianças e adolescentes. Fractal: Revista de Psicologia, 27(2), 139-144. https://doi. org/10.1590/1984-0292/805

Fonseca, J. J. S. (2002). Metodologia da pesquisa científica. UECE. 
Fontes, L. F C., Conceição, O. C., \& Machado, S. (2017). Violência sexual na adolescência, perfil da vítima e impactos sobre a saúde mental. Ciênc. saúde coletiva, 22(9), 2919-2928. https://doi.org/10.1590/1413-81232017229.11042017

Fox, C. L., \& Boulton, M. J. (2005). The social skills problems of victims of bullying: self, peer and teacher perceptions [Os problemas de habilidades sociais das vítimas de bullying: percepção de si mesmo, dos colegas e dos professors]. British Journal of Educational Psychology, 75(2), 313-28. https://doi.org/10.1348/000709905×25517

Habigzang, L. F., Corte, F. D., Hatzenberger, R., Stroeher, F., \& Koller, S. H. (2008). Avaliação psicológica em casos de abuso sexual na infância e adolescência. Psicologia: Reflexão e Crítica, 21(2), 338-344. https://doi.org/10.1590/ S0102-79722008000200021

Instituto Brasileiro de Pesquisa e Estatística. (2016). Pesquisa nacional de saúde do escolar. IBGE. https://biblioteca.ibge. gov.br/visualizacao/livros/liv97870.pdf

Jodelet, D. (2006). Os processos psicossociais da exclusão. In B. Sawaia (Ed.). As artimanhas da exclusão: análise psicossocial e ética da desigualdade social (pp. 53-56). Vozes.

Landini, T. S. (2011). O Professor diante da violência Sexual. Cortez.

Lisboa, C., Wendt, G. W., Neufeld, C. B., \& Matos, M. G. (2014). Satisfação com a vida e com a família e violência interpessoal na adolescência. Revista Brasileira de Terapias Cognitivas, 10(1), 19-28. http://dx.doi.org/10.5935/18085687.20140004

Machado, F. V. (2011). Psicologia social e formação de psicólogos: reflexões a partir de uma experiência docente. Psicologia da Educação, (32), 141-162. http://pepsic.bvsalud.org/scielo.php?script=sci_ arttext\&pid=S1414-69752011000100009

Marra, M. M., \& Costa, L. F. (2018). Entre a revelação e o atendimento: família e abuso sexual. Avances en Psicología Latinoamericana, 36(3), 459-475. https://doi.org/10.12804/ revistas.urosario.edu.co/apl/a.3564

Martins, C. B. G., \& Jorge, M. H. P. M. (2010). Abuso sexual na infância e adolescência: perfil das vítimas e agressores em município do sul do Brasil. Texto \& Contexto - Enfermagem, 19(2), 246-255. https://doi.org/10.1590/S010407072010000200005

Mendes, K. D. S., Silveira, R. C. C. P., \& Galvão, C. M. (2008). Revisão integrativa: método de pesquisa para a incorporação de evidências na saúde e na enfermagem. Texto Contexto Enfermagem, 17(4), 758-64. https://doi.org/10.1590/5010407072008000400018

Miller, J. (2013). The role of sociability self-concept in the relationship between exposure to and concern about aggression in middle school [O papel do autoconceito da sociabilidade na relação entre exposição e preocupação com a agressão no ensino médio]. RMLE Online, 36(7), 1-10. https://www.tandfonline.com/doi/pdf/10.1080/19404 $\underline{476.2013 .11462100}$

Minayo, M. C. S. (2013). O desafio do conhecimento: Pesquisa qualitativa em saúde. Hucitec.

Mitchell, K. J., Jones, L. M., Turner, H. A., Shattuck, A., \& Wolak, J. (2015). The role of technology in peer harassment: Does it amplify harm for youth? [O papel da tecnologia no assédio de colegas: Será que isso amplifica os danos para a juventude?] Psychology of Violence, 6(2), 193-204. https:// psycnet.apa.org/doi/10.1037/a0039317

Miura, P. O., Oliveira, A. S., Galdino, E. T., Santos, K. A. M., Costa, M. L., \& Costa, G. C. (2018). O ambiente escolar como espaço potencial para adolescente: relato de experiência. Pesquisas e Práticas Psicossociais, 13(2), 1-14. http://pepsic. bvsalud.org/scielo.php?script=sci_abstract\&pid=S1809$89082018000200013 \&$ Ing=pt\&nrm=iso

Moher, D., Liberati, A., Tetzlaff, J., \& Altman, D. G. (2009). Preferred Reporting Items for Systematic Reviews and MetaAnalyses: The PRISMA Statement [Itens de Relatório Preferidos para Revisões Sistemáticas e Meta-Análises: A Declaração PRISMA]. PLoS Med 6(7): e1000097. https://doi. org/10.1371/journal.pmed.1000097

Molcho, M., Craig, W., Due, P., Pickett, W., Harel-Fisch, Y., Overpeck, M., \& HBSC Bullying Writing Group (2009). Cross-national time trends in bullying behaviour 1994-2006: findings from Europe and North America [Tendências de tempo internacionais no comportamento de bullying 1994-2006: resultados da Europa e da América do Norte]. International journal of public health, 54 Suppl 2, 225-234. https://doi.org/10.1007/s00038-009-5414-8

Moyano, N., Monge, F. S., \& Sierra, J. C. (2016). Predictors of sexual aggression in adolescents: Gender dominance vs. rape supportive attitudes [Prognósticos de agressão sexual em adolescentes: Dominância de gênero vs. atitudes de apoio ao estupro]. The European of Psychology Applied to Legal Context, 9(1): 25-31. https://doi.org/10.1016/j. ejpal.2016.06.001

Musitu, G., Estévez, E., \& Giménez, T. (2009). Problemas en las aulas: violencia y victimización escolar [Problemas na sala de aula: violência escolar e vitimização]. In R. Landero, M. T. González, B. Estrada, \& G. Musitu (Eds.). Estilos parentales y otros temas en La relación de padres y adolescentes (pp. 191221). Universidad Autónoma de Nuevo León.

Nascimento, A. M. T., \& Menezes, J. A. (2013). Intimidações na adolescência: expressões da violência entre pares na cultura escolar. Psicologia \& Sociedade, 25(1), 142-151. https://doi.org/10.1590/S0102-71822013000100016

Nobre, F., Corrêa, D., Nepomuceno, L., Nobre, L., \& Sousa, A. (2016). A Amostragem na Pesquisa de Natureza Científica 
em um Campo Multiparadigmático: Peculiaridades do Método Qualitativo. Revista Espácios, 38(22), 13. https:// www.revistaespacios.com/a17v38n22/a17v38n21p13.pdf

Oliveira, T., Viana, A. P. S., Boveto, L., \& Sarache, M. V. (2013). Escola, conhecimento e formação de pessoas: considerações históricas. Políticas Educativas, 6(2), 145-160. https://www.seer.ufrgs.br/Poled/article/ download/45662/28843

Oliveira, W. A. D., Silva, J. L. D., Sampaio, J. M. C., \& Silva, M. A. I. (2017). Saúde do escolar: uma revisão integrativa sobre família e bullying. Ciência \& saúde coletiva, 22, 1553-1564. https://doi.org/10.1590/1413-81232017225.09802015

Olweus, D. (1993). Bullying at school: What we know and what we can do [Bullying na escola: O que sabemos e o que podemos fazer]. Wiley-Blackwell.

Olweus, D. (2013). School bullying: Development and some important challenges [Bullying escolar: Desenvolvimento e alguns desafios importantes]. Annual Review of Clinical Psychology, 9(1), 751-780. https://doi.org/10.1146/annurevclinpsy-050212-185516

Pereira, B. O. (2008). Para uma escola sem violência: estudo e prevenção das práticas agressivas entre crianças. Fundação Calouste.

Pigozi, P. L., \& Machado, A. L. (2015). Bullying na adolescência: visão panorâmica no Brasil. Ciência \& Saúde Coletiva 20(11), 3509-3522. https://doi.org/10.1590/1413812320152011.05292014

Platt, V. B., Back, I. C., Hauschild, D. B., \& Guedet, J. M. (2018). Violência sexual contra crianças: autores, vítimas e consequências. Ciência \& Saúde Coletiva, 23(4), 1019-1031. https://doi.org/10.1590/1413-81232018234.11362016

Rajput, M., Goutam, A., \& Rajawat, G. (2018). Bullying and being bullied: prevalence and psychosocial outcomes among school going adolescents of Rohtak [Bullying e intimidação: prevalência e resultados psicossociais entre adolescentes que frequentam a escola de Rohtak]. International Journal of Community Medicine and Public Health, 5, 991-995. https://dx.doi.org/10.18203/2394-6040. ijcmph20180749

Said, A. P., \& Costa, L. F. (2019). Dinâmicas Familiares de Meninos Vítimas de Abuso Sexual. Paidéia (Ribeirão Preto), 29 e2908. https://doi.org/10.1590/1982-4327e2908

Santos, M. J., Mascarenhas, M. D. M., Rodrigues, M. T. P. \& Monteiro, R. A. (2018). Caracterização da violência sexual contra crianças e adolescentes na escola - Brasil, 20102014. Epidemiologia e Serviços de Saúde, 27(2), e2017059. https://doi.org/10.1590/1982-4327e2908

Serafim, A. P., Saffi, F., Achá, M. F. F., \& Barros, D. M. (2011). Dados demográficos, psicológicos e comportamentais de crianças e adolescentes vítimas de abuso sexual. Archives of Clinical Psychiatry (São Paulo), 38, 143-147. https://doi. org/10.1590/S0101-60832011000400006

Sharkey, J. D., Ruderman, M. A., Mayworm, A. M., Green, J. G., Furlong, M. J., Rivera, N., \& Purisch, L. (2015). Psychosocial functioning of bullied youth who adopt versus deny the bully-victim label [Funcionamento psicossocial de jovens maltratados que adotam versus negam o rótulo de bullyvictim]. School psychology quarterly: the official journal of the Division of School Psychology, American Psychological Association, 30(1), 91-104. https://doi.org/10.1037/ spq0000077

Schoeps, K., Villanueva, L., Prado-Gascó, V. J., \& Montoya-Castilla, I. (2018). Development of Emotional Skills in Adolescents to Prevent Cyberbullying and Improve Subjective WellBeing [Desenvolvimento de Habilidades Emocionais em Adolescentes para Prevenir o Cyberbullying e Melhorar o Bem-estar Subjetivo]. Frontiers in psychology, 9, e2050. https://doi.org/10.3389/fpsyg.2018.02050

Silva, R. A., \& Teixeira, L. C. (2017). Adolescência e o traumático: sobre abuso sexual e as vicissitudes do sujeito. Revista Subjetividades, 17(3), 92-103. http://pepsic.bvsalud. org/scielo.php?script=sci_abstract\&pid=\$235907692017000300009\&Ing=pt\&nrm=iso

Souza, J. M. (2013). Bullying: uma das faces do preconceito homofóbico entre jovens no contexto escolar. [Dissertação de Mestrado. Universidade Federal da Sergipe]. Instituto Brasileiro de Informação em Ciência e Tecnologia. https:// bdtd.ibict.br/vufind/Record/UFS-2_6d83553119addc273ad $757 \mathrm{bbdd} 896260$

Souza, M. T., Silva, M. D., \& Carvalho, R. (2010). Revisão integrativa: o que é e como fazer. Einstein, 8, 102-106. https://doi. org/10.1590/S1679-45082010RW1134

Inoue, V. S. R., \& Ristum, M. (2008). Violência sexual: caracterização e análise de casos revelados na escola. Estudos de Psicologia (Campinas), 25(1), 11-21. https://www.scielo.br/j/estpsi/a/ Ryhzvgk9jn3VK9brXPZLDDp/?format=pdf\&lang=pt

World Health Organization. (2003). Guidelines for medico-legal care of victims of sexual violence [Diretrizes para o atendimento médico-legal às vítimas de violência sexual]. World Health Organization. http://www.who.int/violence injuryrevention/ publications/violence/med-legguidelines/en/

Wolff, L. S., Amparo, D. M., Oliveira, R. M., \& Chagnon, J. Y. (2016). Problemática narcísica-identitária em adolescentes abusadores sexuais: contribuições do Rorschach Escola de Paris. Avaliação Psicológica, 15(3), 318-326. http://dx.doi. org/10.15689/ap.2016.1503.04

Zequinão, M. A., Medeiros, P., Pereira, B., \& Cardoso, F. L. (2016). Bullying escolar: um fenômeno multifacetado. Educação e Pesquisa, 42(1), 181-198. https://doi.org/10.1590/S15179702201603138354 